\title{
PULMONARY NON-HODGKIN LYMPHOMA IN A PRIMARY SJÖGREN SYNDROME PATIENT
}

\author{
Claudia Deaconu ${ }^{1}$, Ruxandra Ionescu ${ }^{1,2}$, Daniela Opris ${ }^{1,2}$ \\ ${ }^{1}$ Department of Rheumatology and Internal Medicine, Sfanta Maria Clinical Hospital, Bucharest, Romania \\ ${ }^{2}$ Carol Davila University of Medicine and Pharmacy, Bucharest, Romania
}

\begin{abstract}
Sjögren syndrome (SS) is a chronic autoimmune condition that mainly targets exocrine glands where it produces lymphocytic infiltrates. The clinical display varies in severity and can include malignancy association such as non-Hodgkin lymphoma. Its incidence ranges from 5 to $8 \%$, while the risk of occurrence is 16 to 44 times higher. This article aims to describe the evolution of a patient with primary SS who developed pulmonary lymphoma for which she received Rituximab as part of the oncological therapeutic scheme. During the course of the disease she developed membranous glomerulonephritis, rarely described in these patients and low complement levels cited as predictive factor for lymphoproliferation.
\end{abstract}

Keywords: Sjögren syndrome, non-Hodgkin lymphoma, autoimmune disease

\section{INTRODUCTION}

Sjögren syndrome (SS) is a chronic autoimmune disease characterized by lymphocytic infiltration of exocrine glands, predominantly at salivary and lacrimal sites. This process can present itself alone or be related to other autoimmune disorders (secondary SS) (1). Clinical presentation varies, going from exhibits of decrease in gland secretion consequently to loss of acini and ducts to life-threatening events such as small vessel vasculitis, renal and pulmonary involvement or neoplastic occurrence, primarily malignant non-Hodgkin lymphoma (2). The latter has a $5-8 \%$ incidence, with a 16 to 44 -fold higher risk of development than the general population (3).

\section{CASE PRESENTATION}

We present the case of a 61-year old female patient who was diagnosed with primary SS in 2004 based on the classical triad of xerostomia, xerophalmia and high levels of anti-Ro and anti-La antibodies together with bilateral parotid enlargement and arthralgia of the small joints. Due to the predominance of the articular symptoms, ten milligrams of weekly subcutaneous methotrexate was initiated but discontinued a year later by the patient alone.
The patient was lost to follow-up over a 3-year period when she presented to our clinic with nephrotic syndrome and a 12-gram proteinuria, interpreted at the time as membranous glomerulonephritis considering the clinic-biological features. The display of this extraglandular manifestation was completely remitted under a corticotherapy dose of $1 \mathrm{mg}$ per kg with slow afterward-decrease. Further evolution was favorable on stable treatment with hydroxychloroquine and small dose methylprednisolone.

Ten years from the SS onset, the patient is identified with bilateral axillar adenopathies and a concomitantly rapid decrease in seric complement level. A chest $\mathrm{x}$-ray reveals an inhomogeneous pulmonary opacity in the right medium lobe (RML). A computed tomography examination was performed, showing multiple iodophilic lymph nodes and a relatively well-defined tissue infiltration measuring $22 / 18 \mathrm{~mm}$ in the medial segment of the RML, with no visible obstruction or other organ involvement on the examined slices.

The hematological check up at the time showed no peripheral discharge that could have allowed imunophenotyping, hence a pneumological examination was recommended. The bronchoscopy found

Correspondence address:

Claudia Deaconu, Department of Rheumatology and Internal Medicine, Sf. Maria Clinical Hospital, 37-39 Ion Mihalache Bd, District 1,

Bucharest

E-mail: claudiadeaconu1@yahoo.com 
no lesions and the bronchoalveolar lavage unveiled an increased cellularity $(23 / \mathrm{ml})$ with pronounced lymphocytosis (44\%) but with no tumor cells.

A second CT scan confirmed a slight enlargement of the pulmonary condensation with a regression in adenopathic dimensions, otherwise a stationary imagistic aspect.

Taking into account the progression of the pulmonary mass of unknown origin, a medium lobe lobectomy is undergone with subsequent histopathologic examination. Macroscopic findings were that of a white-grey tumor formation of 3.5/1.8/4 cm with defined margins with a tendency at retracting the adjacent pleura. Microscopically, the pulmonary parenchyma was densely and diffusely infiltrated with large cells that destroyed the normal architecture, therefore orienting to a diffuse large cell lymphoma.

Further immunohistochemistry (IHC) testing detected that the nodular lymphoid infiltrates contained small cells that were CD20 positive and expressed Cyclin D1 which is the mantle cell lymphoma marker. The kappa/lambda ratio of $1 / 10$ proved its clonal

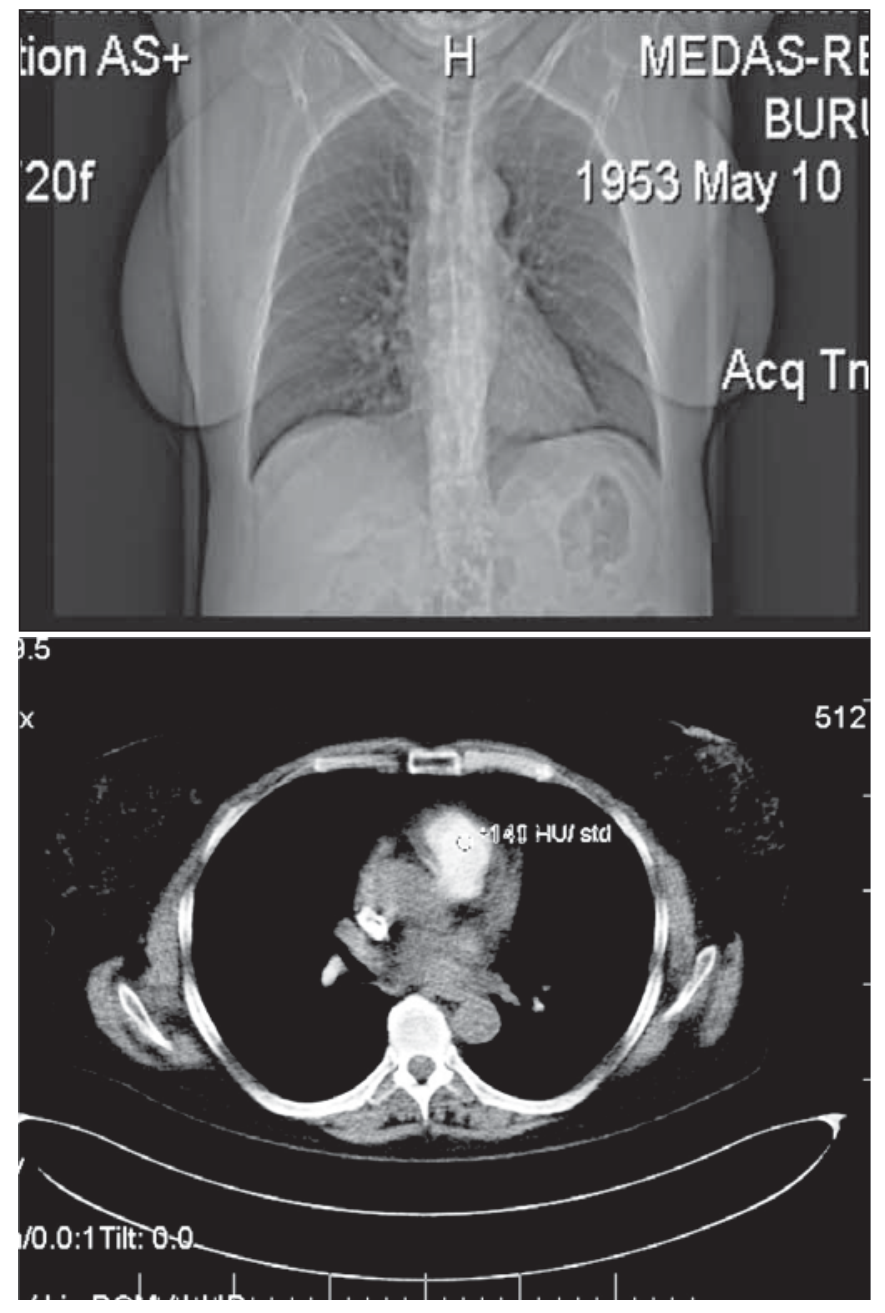

origin and the cells were negative for $\mathrm{T}$ and centrofollicular markers such as CD5, CD 10 and BCL6. The histopathological together with IHC testing were compatible with malignant non-Hodgkin small B cell mantle lymphoma.

Following diagnosis confirmation the patient was admitted to the Hematology Department in order to fulfill a complete evaluation prior to prescribing a chemotherapy appropriate scheme. The biological parameters showed no abnormalities and the myelogram interpretation was that of present maturation of all cellular series with $20 \%$ small lymphocytes. The bone marrow biopsy confirmed the absence of malignant lymphoid infiltrates at this level. The patient was negative for HIV, hepatic B and C viral infection. The abdominal ultrasound as well as the tomographic imaging showed no suggestive signs of secondary site determination. The echocardiography was within normal range.

The pretherapeutic medical scrutiny concurred that the stage IV lymphoma would better respond to a CHOP chemotherapy scheme as follows: cyclophosphamide $1350 \mathrm{mg}$, doxorubicine $90 \mathrm{mg}$, vin-

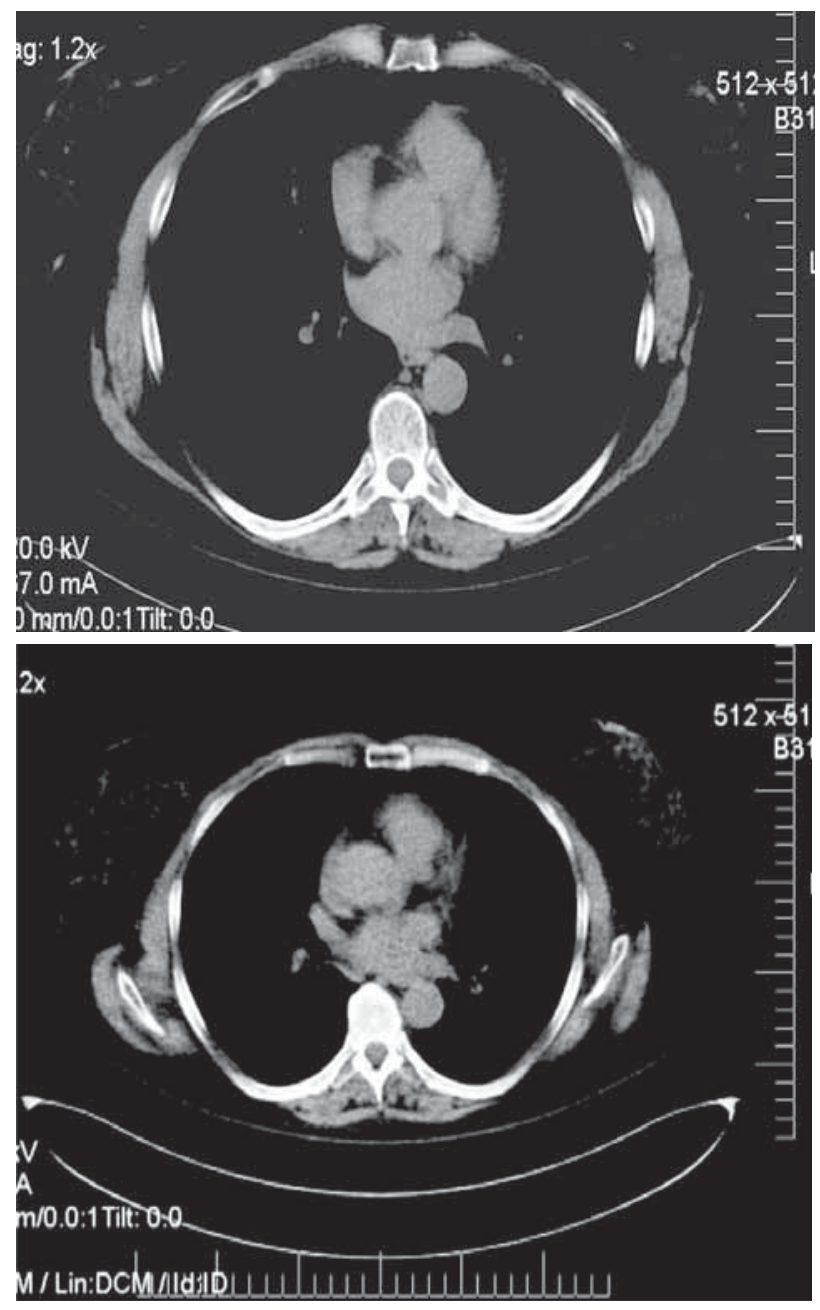


cristine $2 \mathrm{mg}$ on day 1 and dexamethasone $16 \mathrm{mg}$ from day 1 to day 5 .

With the third cycle, rituximab $700 \mathrm{mg}$ together with its correspondent premedication was added to the therapeutic strategy.

The patient needed close monitoring when undergoing chemotherapy cures as she presented with low level potassium (as much as $2.6 \mathrm{mmol} / \mathrm{l}$ at the latest evaluation) that expresses through symptoms such as weakness, fatigue, muscle cramps and cardiac discomfort due to arrhythmias.

\section{DISCUSSIONS}

In recent years increasing data draws attention upon the association of autoimmune diseases and malignant lymphoproliferative processes. The most frequent correlations were made with Sjögren syndrome, autoimmune thyroiditis and autoimmune hemolytic anemia. This liaison has been rarely cited for rheumatoid arthritis (RA) (4).

A meta-analysis using the PubMed database from 1974 to 2005, published by Zintzaras et al. who followed the association between non-Hodgkin malignant lymphoma and autoimmune conditions showed an increased risk for SS, a moderate risk for systemic lupus erythematosus (SLE) and a low hazard rate for RA (5). Similar data was found by a multicentric, multinational study that included an impressive number of patients with autoimmune disorders (29 493) or by a Scandinavian study who was interested in the same theory (6).

Sjögren syndrome best illustrates the link between autoimmunity and lymphoproliferation (7). This incidence is among the highest ever quoted for autoimmune diseases, with an estimated relative risk of 6.1, according to the above-mentioned Scandinavian study.

Primary SS is an autoimmune inflammatory condition associated with the production of autoantibodies that target the exocrine glands, having a chronic and relatively silent evolution. In spite its apparently benign course SS is associated with a high risk of malignant lymphoma, which was first detected by Bunim and Talal in 1963 (8).

The autoimmune disease usually precedes the malignant transformation with a mean period of 7.5 years but literature cites certain case reports with intervals as long as 30 years (9). Due to the fact that the SS diagnosis is established with a certain delay when sicca symptoms are extensive, it is quite diffi- cult to establish the evolution interval of the autoimmune process compared to the hematological one.

Histologically lymphomas belong most frequently to the marginal type subgroup. These originate in the B lymphocyte while the $\mathrm{B}$ cell population is only $20 \%$ if the total of the infiltrate. Diffuse large cell lymphomas, follicular or immunoblastic lymphomas have also been reported, with an unfavorable outcome $(4,10)$.

The lymphocytic transformation frequently occurs at the active sites such as salivary glands but they can also have other targets (stomach, pharynx, skin, renal or pulmonary) (10).

Together with the genetic susceptibility and the environmental factors the pathogenesis of SS is dominated by large lymphoid infiltrates that form masses which are called benign lympho-epithelial lesions. Future transition to malignant lymphoma is a process with no fully understood mechanism, having more steps driven by chronic antigen stimulation.

Continuous stimulation of reactive B cells in the affected organs can explain the lymphomatous proliferation at this site. Certain defects in B cells apoptosis together with structural alterations can also be involved (e.g. BlyS anti-apoptotic cytokine that stimulates the clonal expansion of B lymphocytes).

A Swedish study on a cohort of 175 patients with SS had a biopsy taken from the salivary tissue and identified lymphoid groups with a germinative center (GC) structure which proved to be highly predictive for further non-Hodgkin lymphoma. These GC forms were detected in $30 \%$ of patients and they were associated with the presence of extraglandular manifestations, high rheumatoid factor titers and the presence of anti-nuclear antibodies.

The need to identify predictive factors of progression in SS led to numerous studies but at present there is no unanimous consensus on this topic. However we know that the investigations made at the moment of the diagnosis have prognostic value for further malignant transformation. A recently published meta-analysis estimated that the risk of lymphoma and lymphoproliferative disease is around $4 \%$ of patients in the first five years, $10 \%$ at 15 years and $18 \%$ at 20 years from the moment of diagnosis. This study pointed out the main progression predictors, including parotid enlargement, suggesting a high lymphoid reactivity. It is still unclear if this is a predictive factor or a red flag for an already settled lymphoma. Splenomegaly, persistent lymphadenop- 
athy, palpable purpura, arthralgia and neuropathy are associated to various extents with neoplastic development. Moreover, associating both vasculitis with low serum complement levels indicates a high risk of malignant transformation, according to Ioannidis et al. in 2002.

Among the serological parameters that were linked to lymphoma in SS patients, the presence of cryoglobulins, lymphopenia, neutropenia and a low level of $\mathrm{C} 3$ complement fragment are highly suggestive for malignant progression. Low level of $\mathrm{C} 4$ has been identified as predictive for lymphoproliferation but also for global survival of most patients (11).

According to the most recent meta-analysis, anemia, positive rheumatoid factor of anti-nuclear antibodies and hypergammaglobulinemia were not mentioned as predictors for the neoplastic proliferation(5).

Another study in 2013 that included 195 patients mentioned glomerulonephritis as a paraneoplastic manifestation and not a risk factor for lymphoma (12). Moreover, a recent study centered on renal biopsies in patients with primary SS suggest that glomerulonephritis and not tubulo-interstitial nephritis is the one associated with a higher rate of mortality and a reduction of up to three years in patient survival (66 versus $100 \%, \mathrm{P}=0.02$ ) (13). As seen in our patient she presented with glomerulonephritis which was rapidly responsive to corticotherapy but at the time she had no other serological or pulmonary abnormalities that could have oriented us to the diagnosis.

The presence of more risk factors, both clinical and serological increases the possibility of further malignant transformation and this risk increases with disease duration. Patients that develop these risk factors should be regularly monitored.

Therapeutic options for SS are at the moment a matter of debate. Taking into consideration the major role of B cells in the pathophysiology of SS, their depletion appears to be a rational approach for testing.

Rituximab has proven its efficacy in patients with primary SS that associate systemic manifestations and studies have shown a significant reduction in the ESSDAI (European Sjögren's Syndrome Disease Activity Index) score together with a lower dose of corticotherapy(14). In the study of Dass et al. (2008) which included 18 patients indicate a positive effect on the VAS for fatigue after 6 months of treatment (15) while Meijer et al. showed an improvement in the dryness VAS and the salivary flow rate at 6 months (16). The TRACTISS study which aims at evaluating the role of Rituximab on clinical symptoms is still ongoing (17).

Interestingly, Rituximab showed a reduction in the glandular infiltrate and limited the formation of lymphoid ectopic structures and GC (18).

Patients with SS have high levels of BAFF/Blys (B-cell activating factor/B lymphocite stimulator) in their serum and salivary glands (19). An excessive expression of BAFF might explain the characteristic activation of B lymphocites in primary SS (20).

A phase II trial (BELISS study) using Belimum$\mathrm{ab}$ in patients with SS proved a reduction in sicca symptoms and the VAS (Visual Analogue Scale) parameters for pain and fatigue, with future promising results (21).

The open label proof of concept ASAP study issued in 2014 used Abatacept in 15 early and active primary SS patients (22). Abatacept is a fusion molecule of Fc IgG and CTLA 4 (cytotoxic T lymphocite antigen 4) that inhibits CD28 mediated T cell stimulation. This study showed a reduction in the ESSDAI score at week 24 of treatment $(p<0.001)$ mostly in the articular, biological and glandular fields. There was also an improvement in pain and fatigue. The treatment was overall well tolerated, with no severe adverse events (23).

Lymphoma is the main cause of death in SS patients with a risk rate of 2.5 in 1000 patients-years (7). Advanced stages, large scaled tumors especially over $7 \mathrm{~cm}$ and large cell lymphoma are unfavorable prognostic factors, not only through concomitant complications but also through their unsatisfactory response to treatment (6). Mean survival rate in these patients is of approximately two years $(1,7)$.

\section{CONCLUSIONS}

Non-Hodgkin lymphoma is the most fearful complication in patients with SS, with huge impact on their prognostic and survival. Identifying clinical and serological predictive factors can help to promptly detect malignant transformation. Patients at high risk should be closely followed so that the treatment is rapidly installed in case of neoplastic occurrence. 


\section{REFERENCES}

1. P. Youinou, J.-O. Pers, Primary Sjögren's syndrome at a glance today, Jt. Bone Spine, vol. 82, no. 2, pp. 75-76, Mar. 2015.

2. A. Tincani, L. Andreoli, I. Cavazzana, A. Doria, M. Favero, M.G. Fenini, F. Franceschini, A. Lojacono, G. Nascimbeni, A. Santoro, F. Semeraro, P. Toniati, Y. Shoenfeld, Novel aspects of Sjogren's syndrome in 2012, BMC Med, vol. 11, p. 93, 2013.

3. M. Ramos-Casals, P. Brito-Zerón, A. Sisó-Almirall, X. Bosch, Primary Sjogren syndrome, BMJ, vol. 344, p. e3821, 2012.

4. K.E. Smedby, H. Hjalgrim, J. Askling, E.T. Chang, H. Gregersen, A. Porwit-MacDonald, C. Sundström, M. Akerman, M. Melbye, B. Glimelius, H.O. Adami, Autoimmune and chronic inflammatory disorders and risk of non-Hodgkin lymphoma by subtype, J. Natl. Cancer Inst., vol. 98, no. 1, pp. 51-60, Jan. 2006.

5. E. Zintzaras, M. Voulgarelis, H.M. Moutsopoulos, The risk of lymphoma development in autoimmune diseases: a meta-analysis, Arch. Intern. Med., vol. 165, no. 20, pp. 2337-44, Nov. 2005.

6. S.J. Johnsen, J.G. Brun, L.G. Gøransson, M.C. Småstuen, T.B. Johannesen, K. Haldorsen, E. Harboe, R. Jonsson, P.A Meyer, R. Omdal, Risk of non-Hodgkin's lymphoma in primary Sjögren's syndrome: a population-based study, Arthritis Care Res. (Hoboken)., vol. 65, no. 5, pp. 816-21, May 2013.

7. I.F. Horvath, A. Szanto, G. Papp, M. Zeher, I.F. Horvath, A. Szanto, G. Papp, M. Zeher, Clinical Course, Prognosis, and Cause of Death in Primary Sjögren's Syndrome, J. Immunol. Res., vol. 2014, pp. 1-8, 2014

8. P. Thomas, Sjögren's syndrome, dysimmunoglobulinaemia and malignant disease, Postgrad. Med. J., vol. 49, no. 571, pp. 349-54, May 1973

9. M. Kauppi, E. Pukkala, H. Isomäki, Elevated incidence of hematologic malignancies in patients with Sjögren's syndrome compared with patients with rheumatoid arthritis (Finland), Cancer Causes Control, vol. 8, no. 2, pp. 201-4, Mar. 1997.

10. E. Theander, G. Henriksson, O. Ljungberg, T. Mandl, R. Manthorpe, L.T.H. Jacobsson, Lymphoma and other malignancies in primary Sjogren's syndrome: a cohort study on cancer incidence and lymphoma predictors, Ann. Rheum. Dis., vol. 65 , no. 6, pp. 796-803, Jun. 2006

11. S.S. Kassan, T.L. Thomas, H.M. Moutsopoulos, R. Hoover, R.P. Kimberly, D.R. Budman, J. Costa, J.L. Decker, T.M. Chused, Increased risk of lymphoma in sicca syndrome, Ann. Intern. Med., vol. 89, no. 6, pp. 888-92, Dec. 1978.

12. A.V. Goules, I.P. Tatouli, H.M. Moutsopoulos, A.G. Tzioufas, Clinically significant renal involvement in primary Sjögren's syndrome: clinical presentation and outcome, Arthritis Rheum., vol. 65 , no. 11, pp. 2945-53, Nov. 2013.

13. D. Kidder, E. Rutherford, D. Kipgen, S. Fleming, C. Geddes, G.A. Stewart, Kidney biopsy findings in primary Sjögren syndrome, Nephrol. Dial. Transplant, vol. 30, no. 8, pp. 1363-9, Aug. 2015.

14. J. Pijpe, G.W. Van Imhoff, A. Vissink, J.E. Van Der Wal, P.M. Kluin, F.K.L. Spijkervet, G.M. Kallenberg, H. Bootsma, Changes in salivary gland immunohistology and function after rituximab monotherapy in a patient with Sjögren's syndrome and associated MALT lymphoma, Ann Rheum Dis, vol. 64, pp. 958-960, 2005.

15. S. Dass, S.J. Bowman, E.M. Vital, K. Ikeda, C.T. Pease, J. Hamburger, A. Richards, S. Rauz, P. Emery, Reduction of fatigue in Sjögren syndrome with rituximab: results of a randomised, double-blind, placebo-controlled pilot study, Ann. Rheum. Dis., vol. 67, no. 11, pp. 1541-4, Nov. 2008.

16. J.M. Meijer, P.M. Meiners, A. Vissink, F.K.L. Spijkervet, W. Abdulahad, N. Kamminga, E. Brouwer, C.G.M. Kallenberg, $\mathrm{H}$. Bootsma, Effectiveness of rituximab treatment in primary Sjögren's syndrome: a randomized, double-blind, placebocontrolled trial, Arthritis Rheum., vol. 62, no. 4, pp. 960-8, Apr. 2010.

17. S. Brown, N. Navarro Coy, C. Pitzalis, P. Emery, S. Pavitt, J. Gray, C. Hulme, F. Hall, R. Busch, P. Smith, L. Dawson, M. Bombardieri, N. Wan-Fai, C. Pease, E. Price, N. Sutcliffe, C. Woods, S. Ruddock, C. Everett, C. Reynolds, E. Skinner, A. Poveda-Gallego, J. Rout, I. Macleod, S. Rauz, S. Bowman, TRACTISS trial team, The TRACTISS protocol: a randomised double blind placebo controlled clinical trial of anti-B-cell therapy in patients with primary Sjögren's Syndrome, BMC Musculoskelet. Disord., vol. 15, p. 21, 2014.

18. F. Carubbi, P. Cipriani, A. Marrelli, P. Benedetto, P. Ruscitti, O. Berardicurti, I. Pantano, V. Liakouli, S. Alvaro, A. Alunno, A. Manzo, F. Ciccia, R. Gerli, G. Triolo, R. Giacomelli, Efficacy and safety of rituximab treatment in early primary Sjögren's syndrome: a prospective, multi-center, follow-up study, Arthritis Res. Ther., vol. 15, no. 5, p. R172, 2013.

19. F. Lavie, C. Miceli-Richard, J. Quillard, S. Roux, P. Leclerc, X. Mariette, Expression of BAFF (BLyS) in T cells infiltrating labial salivary glands from patients with Sjögren's syndrome, J. Pathol., vol. 202, no. 4, pp. 496-502, Apr. 2004.

20. J.O. Pers, C. Daridon, V. Devauchelle, S. Jousse, A. Saraux, C. Jamin, P. Youinou, BAFF overexpression is associated with autoantibody production in autoimmune diseases, Ann. N. Y. Acad. Sci., vol. 1050, pp. 34-9, Jun. 2005.

21. X. Mariette, S. Roux, J. Zhang, D. Bengoufa, F. Lavie, T. Zhou, R. Kimberly, The level of BLyS (BAFF) correlates with the titre of autoantibodies in human Sjögren's syndrome, Ann. Rheum. Dis., vol. 62, no. 2, pp. 168-71, Feb. 2003.

22. H. Yin, C.Q. Nguyen, Y. Samuni, T. Uede, A.B. Peck, J.A. Chiorini, Local delivery of AAV2-CTLA4IgG decreases sialadenitis and improves gland function in the C57BL/6.NODAec1Aec2 mouse model of Sjögren's syndrome, Arthritis Res. Ther., vol. 14, no. 1, p. R40, 2012.

23. P.M. Meiners, A. Vissink, F.G.M. Kroese, F.K.L. Spijkervet, N.S. Smitt-Kamminga, W.H. Abdulahad, J. Bulthuis-Kuiper, E. Brouwer, S. Arends, H. Bootsma, Abatacept treatment reduces disease activity in early primary Sjögren's syndrome (open-label proof of concept ASAP study), Ann. Rheum. Dis., vol. 73, no. 7, pp. 1393-6, Jul. 2014. 